Figure S1-A

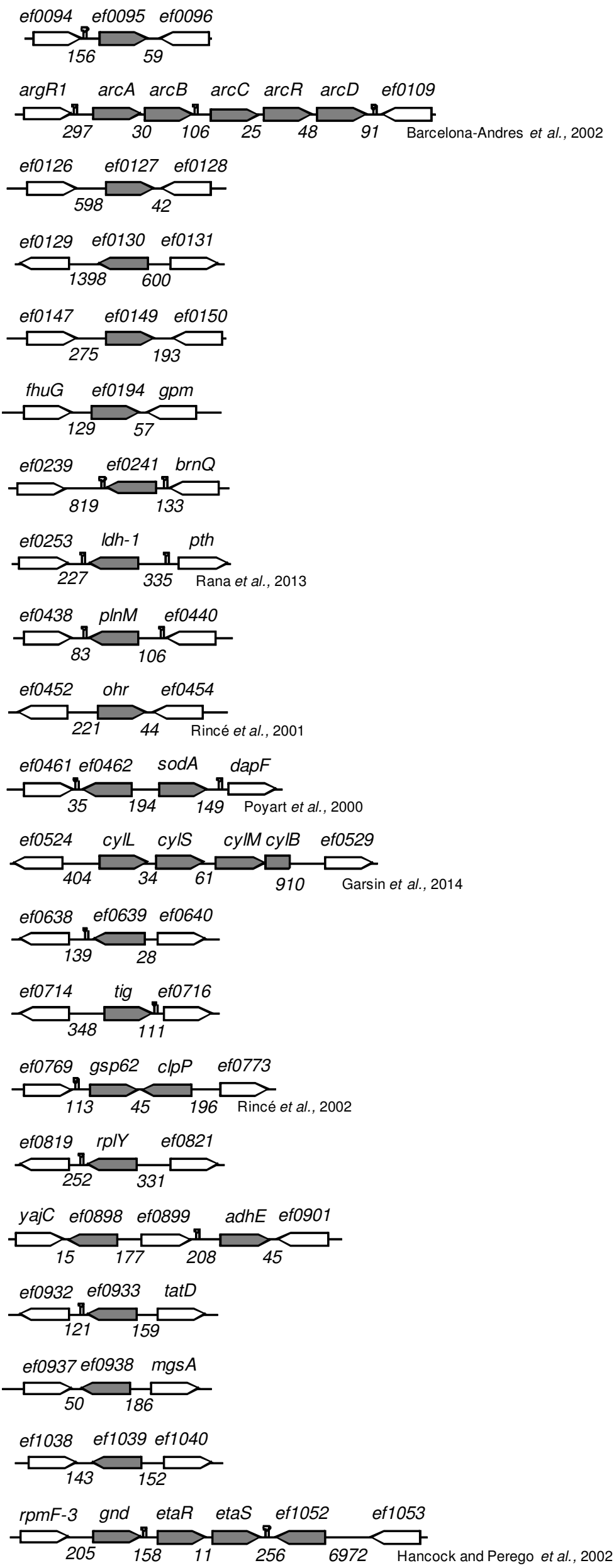




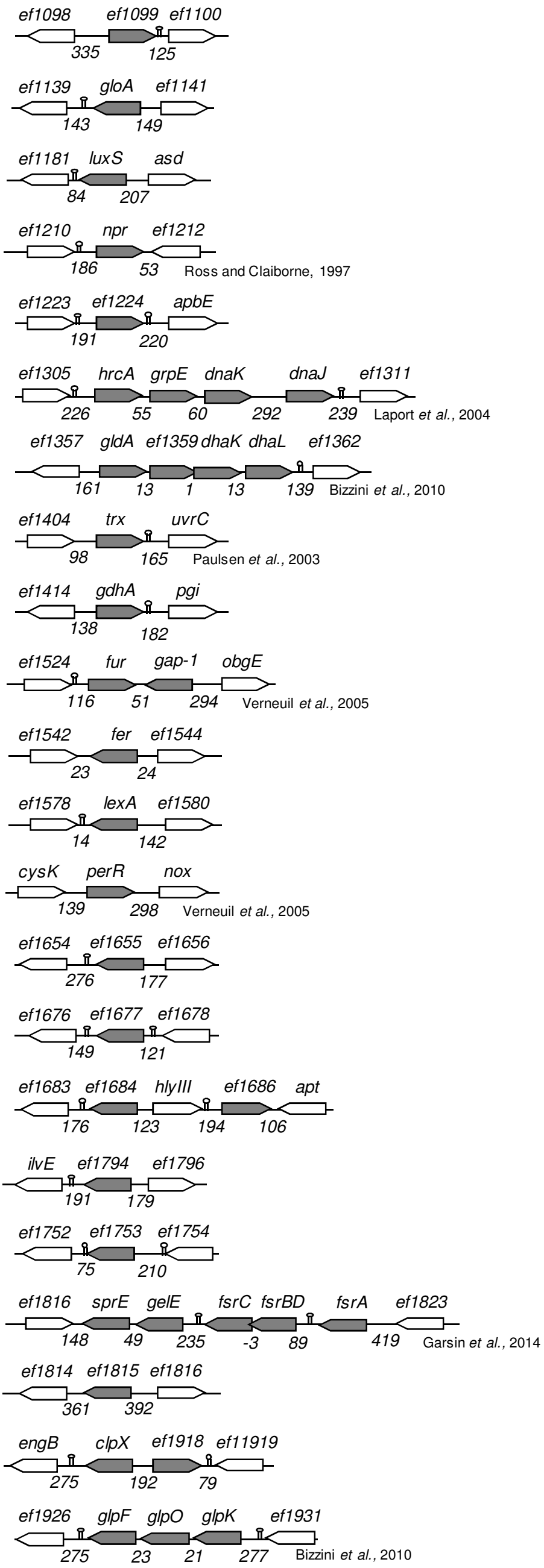


prsA-1 efaC efaB efaA ef2077

$r_{-314}{ }_{-3}$

$\underbrace{\text { ef2354 clpB ef2357 }}_{35}$

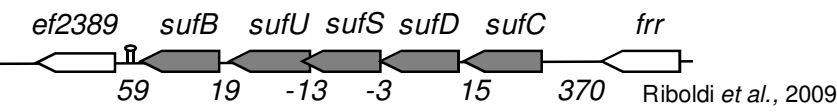

ef2469 ef2470 args

$\longrightarrow$

ef2479 ef2480 ef2481

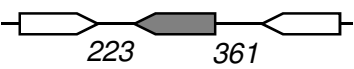

ef2582 ef2583 ef2584

404

ef2632 groEL groES ef2636

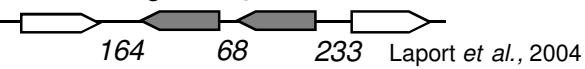

ef2666 ef2667 mgtE

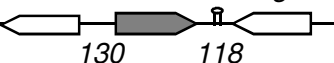

ef2675 mecA ef2678

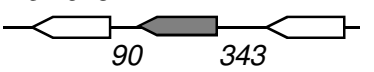

ef2696 ef2697 ef2698 ef2699

$\longrightarrow \longrightarrow_{20}$

ef2712 ef2713 rpll

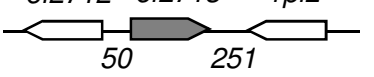

ef2731 ef2732 ef2733

$\longrightarrow_{286}^{\longrightarrow} \longrightarrow$

ef2736 ahpF ahpC ef2740

$\longrightarrow 812$ (D2

ef2900 ef2901 ef2902

$\overbrace{-12}^{\longrightarrow}{ }_{65}^{+}$

ef2938 ef2939 ef2940

$\longrightarrow 204 \longrightarrow$

ef3087 ef3088 ef3089

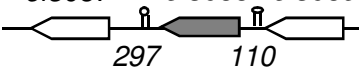

ef3151 mscL ef3153

$\overbrace{209}^{\longrightarrow}$

ef3177 ef3178 ef3179

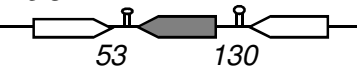

ef3205 ef3206 ef3207

$-\square$

apbE ef3256 ef3257

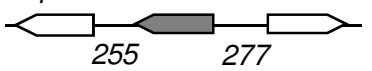

ef3287 croR cros serS-2

$225-10 \quad 335$ Hancock and Perego, 2002 


\section{Figure S1-B}
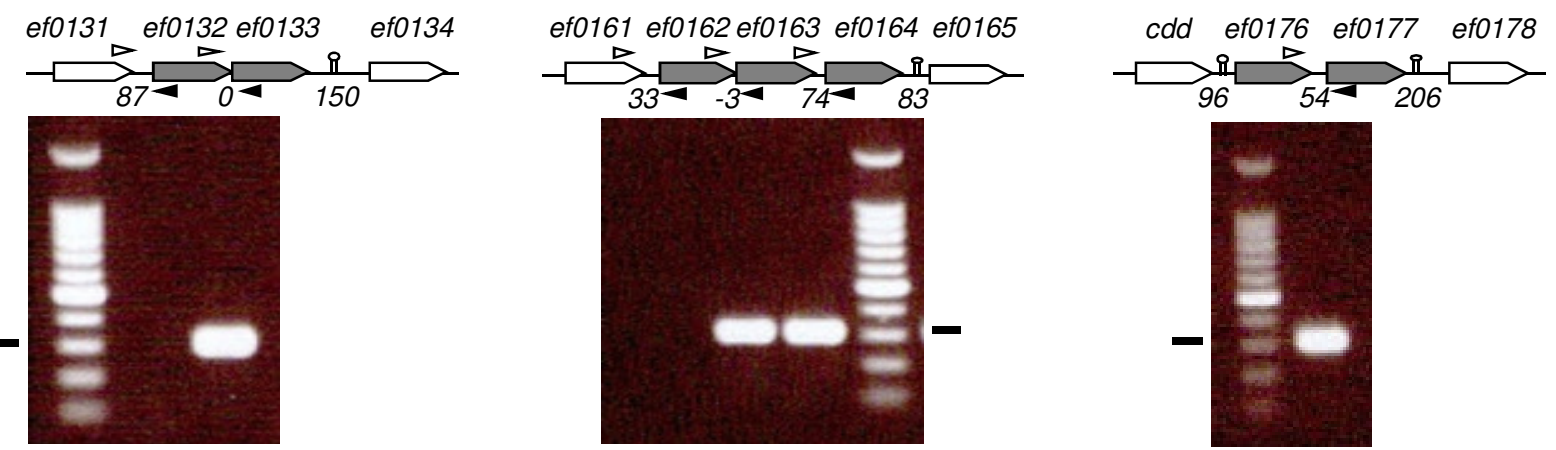

ef0184 deoB deoD-1 deoD-2
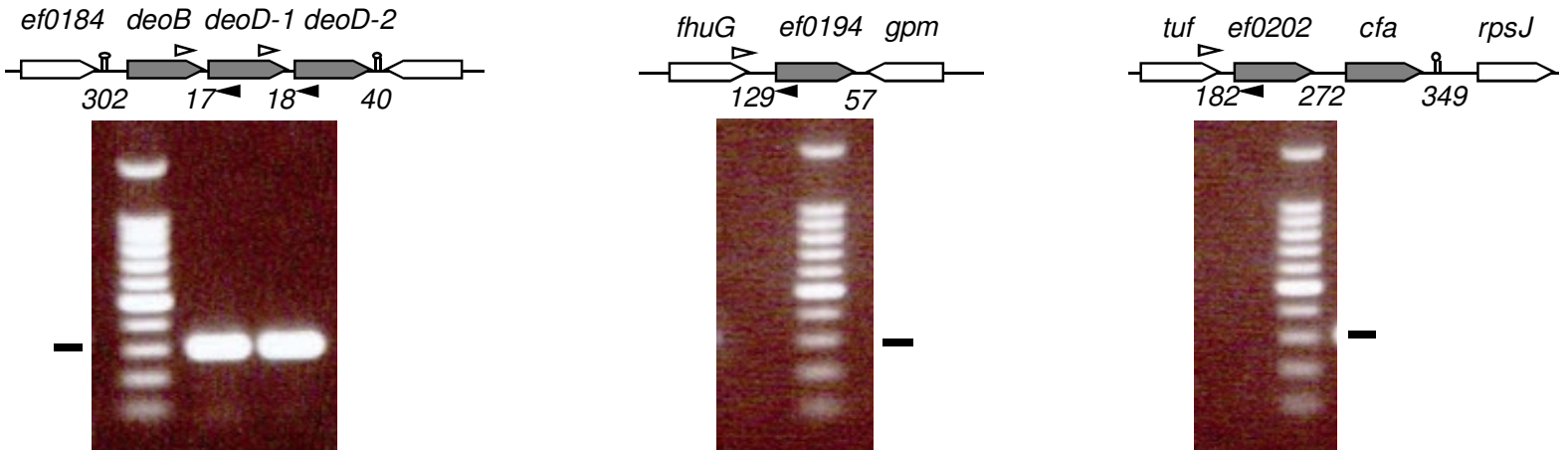

fabl fabF-1 fabZ-1 pyrD-1 ef0286 efp ef0288 cysB metC celA ef0292 ef0293 ef0331 ef0332 ef0333 ef0334 ef0335 ef0336 ef0337

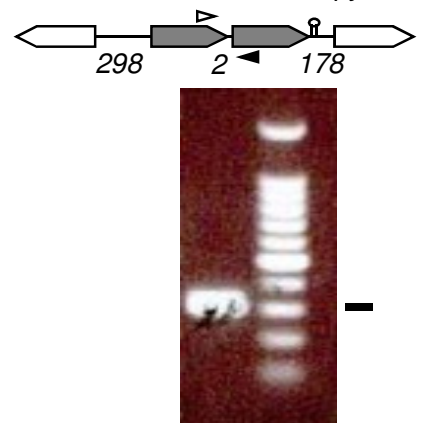

sugE-2 ef0361 ef0362 ef0363

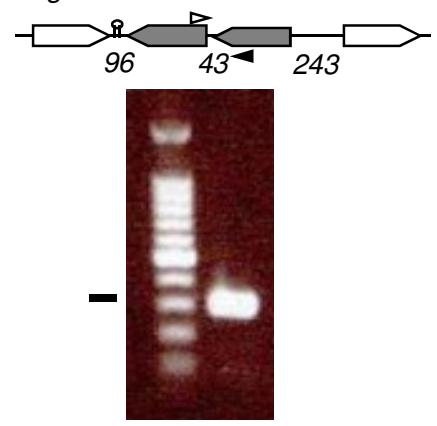

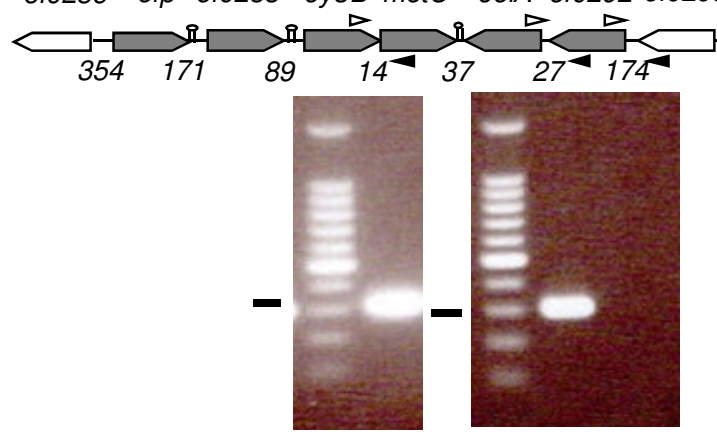

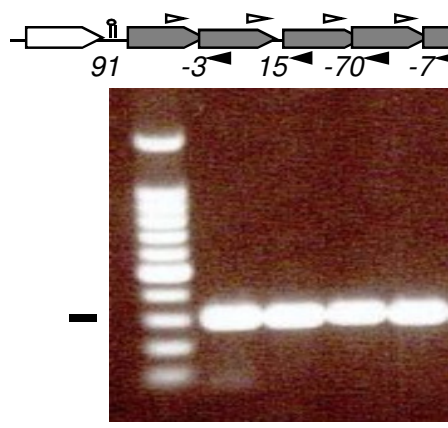

ef0376 ef0377 ef0378 ef0379

ef0574 ef0575 ef0576 ef0577ef0578 ef0579 ef0580

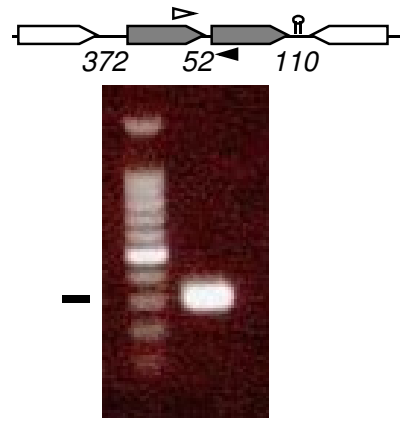

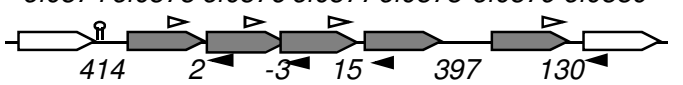

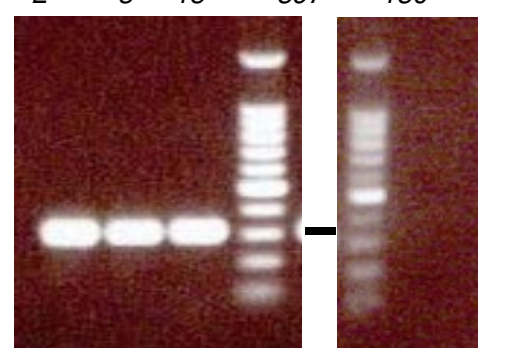

ef0599 ef0600 ef0601 ef0602

tryS-1 ef0634 ef0635 nhaC-2 ef0637
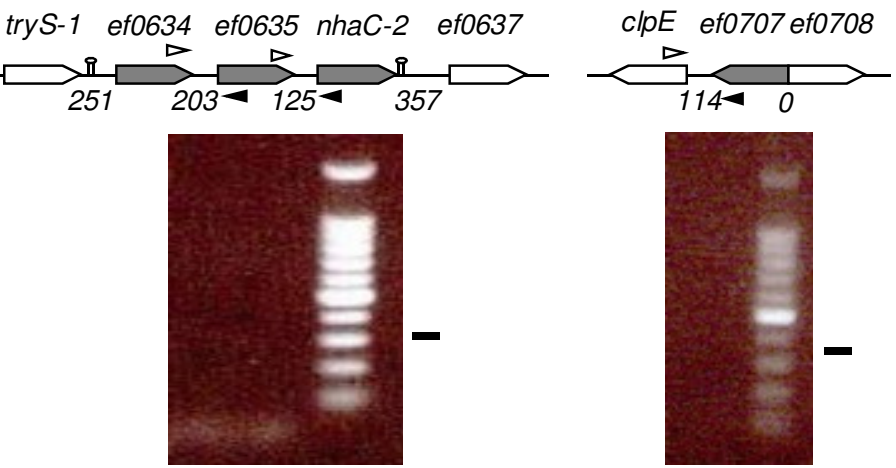

ef0746 ef0747 ef0748
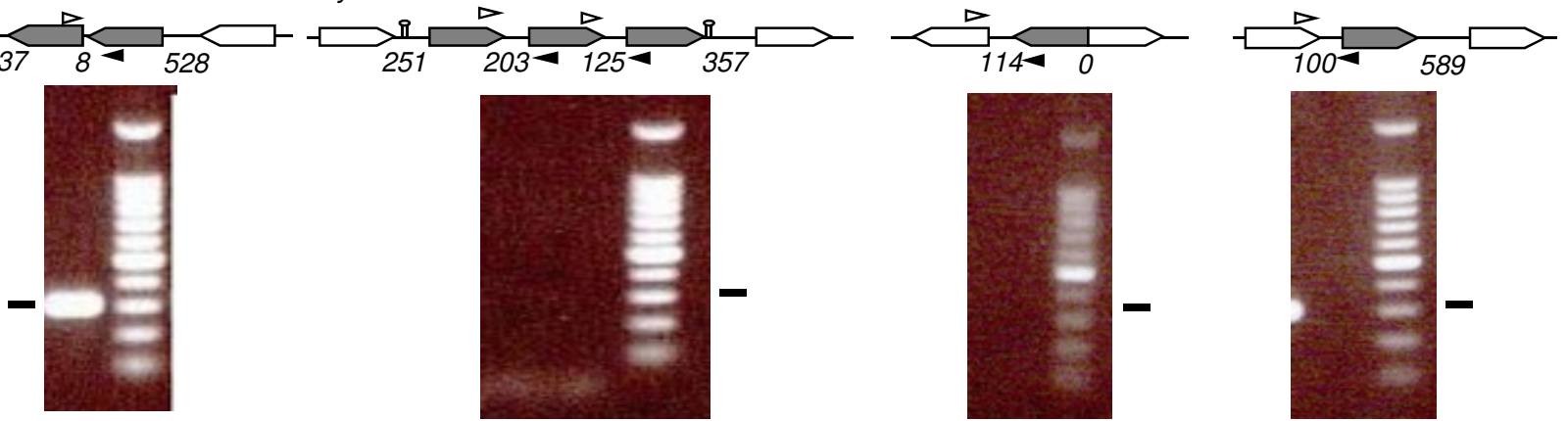

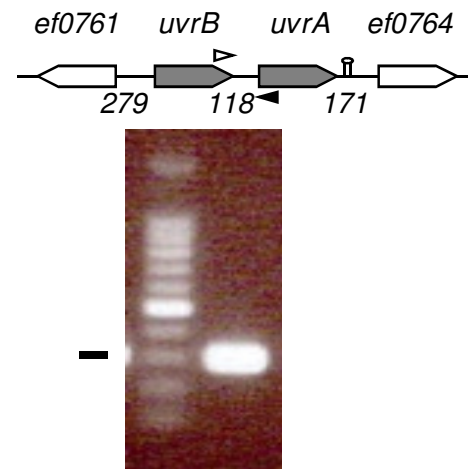

ef1056 ef1057 ef1058 ef1059
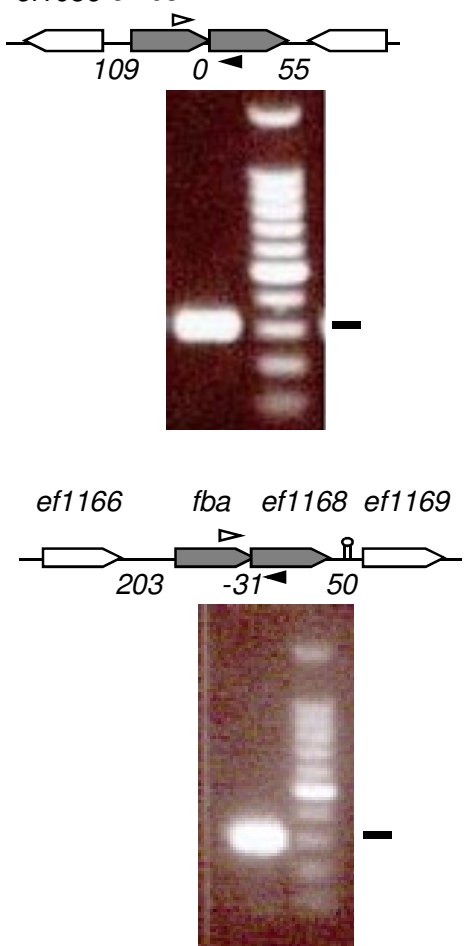

alaS ef1380 ef1381 pepT-1 ef1383
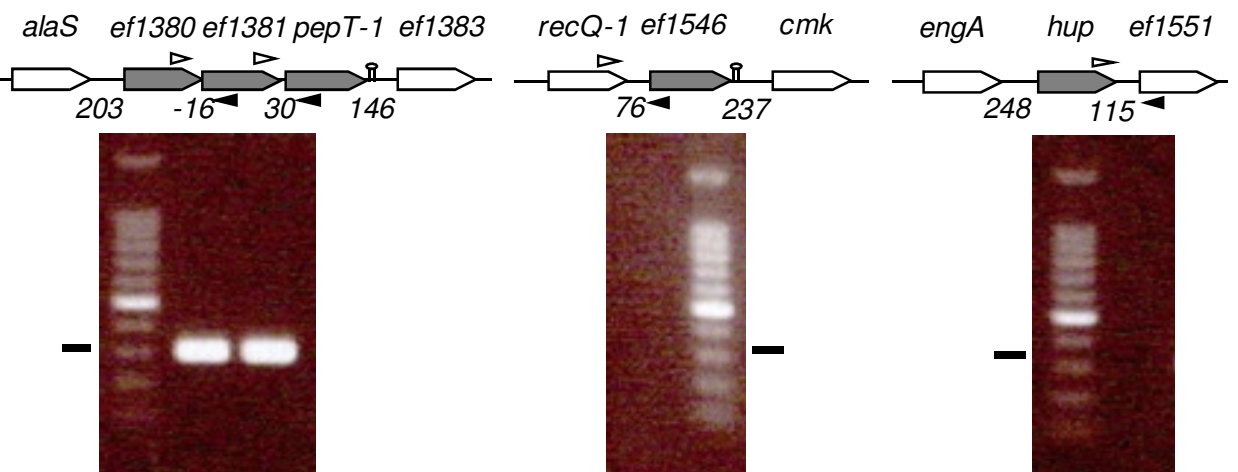

ef1106 ef1107 ef1108 ef1109 ef1110 ef1111
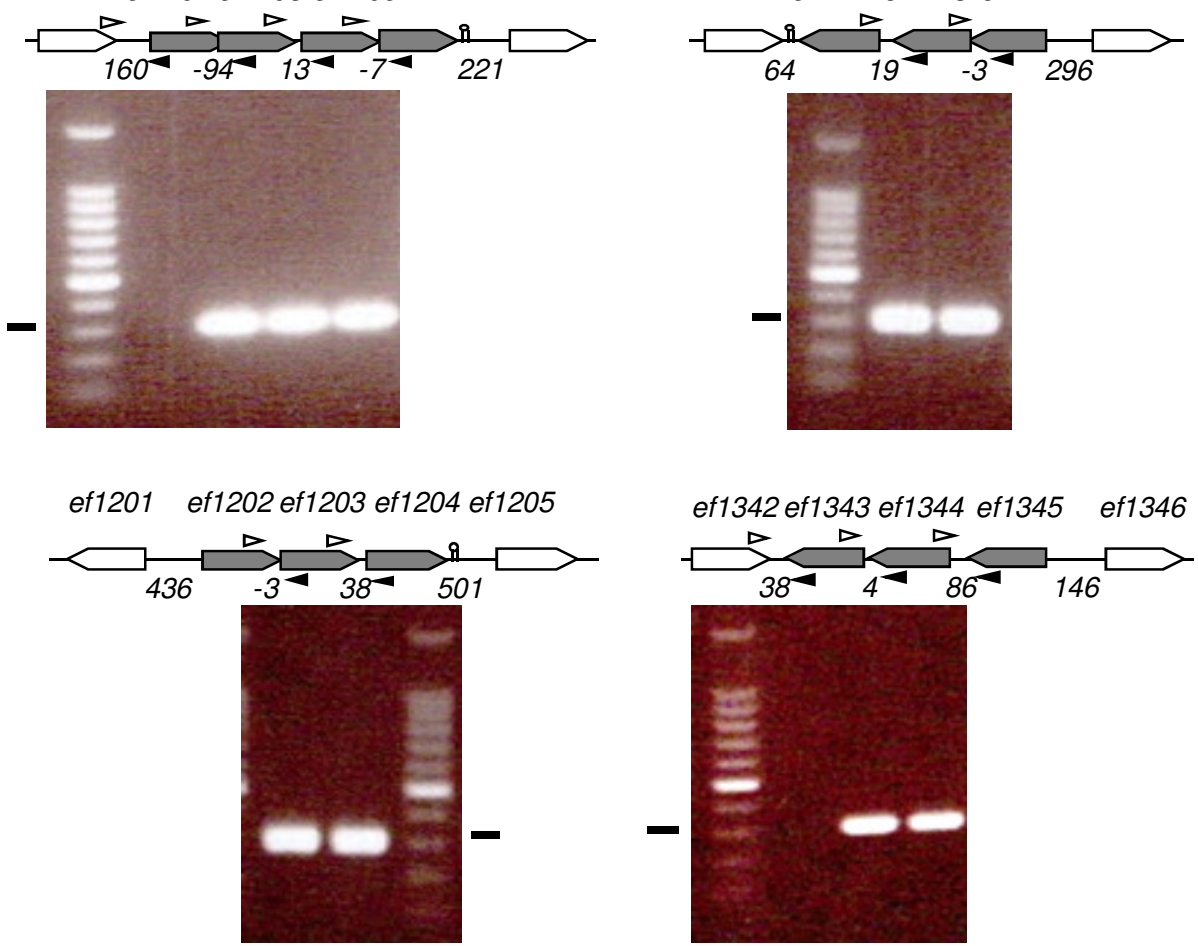

ef0945 ef0946 ef0947

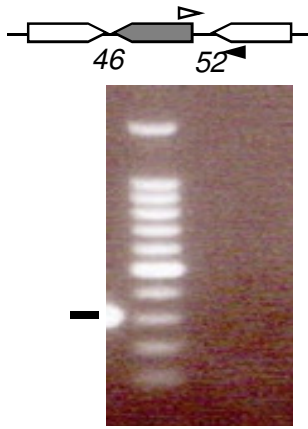

ef1141 ef1142 ef1143 ef1144 ef1145
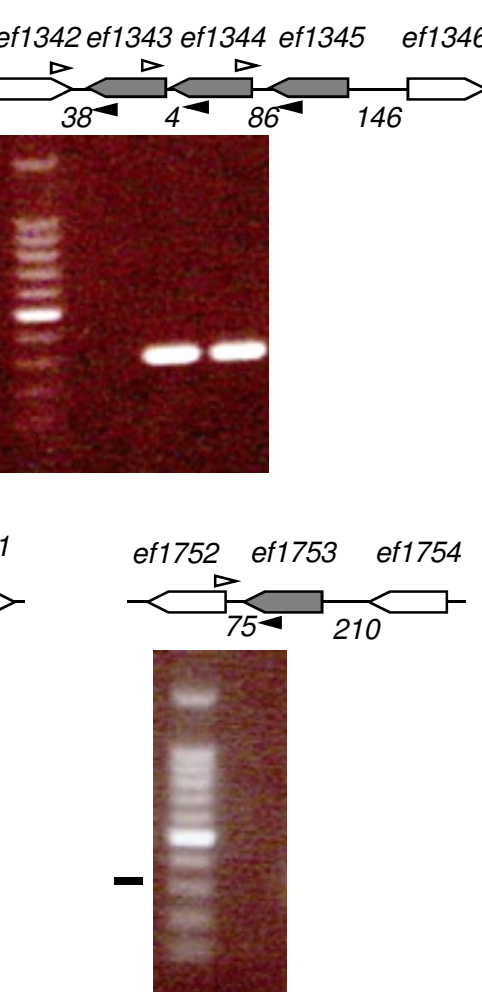

ef1657 bkdC bkdB bkdA bkdD buk ptb ef1664

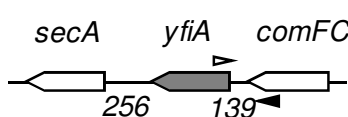

ef1768 ef1769 ef1770
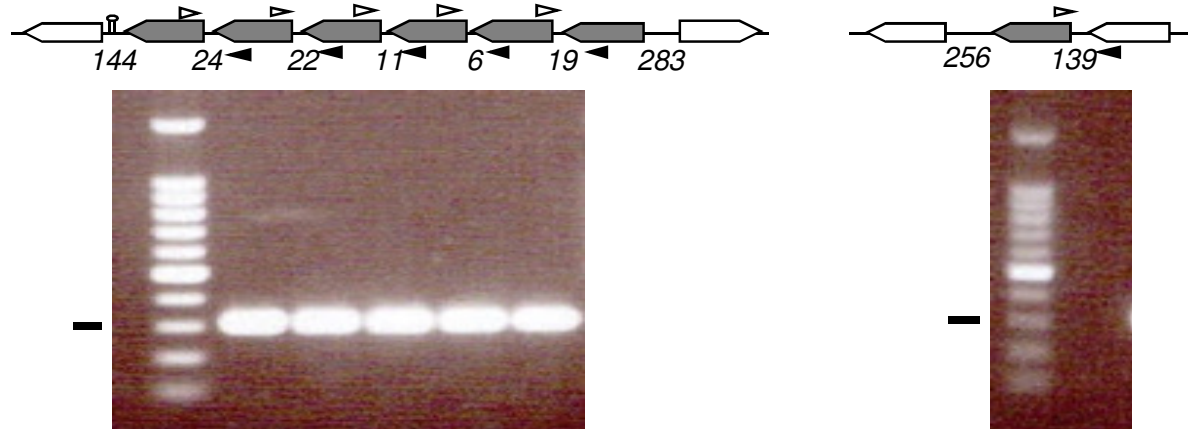
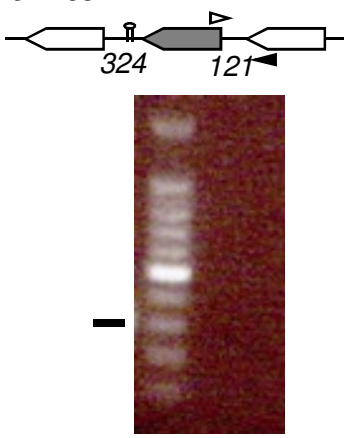
Figure S1-B

ef1866 ef1867 ef1868 ef1869 ef1870 ef1941 ef1943 ef1944 ef1966 ef1967 ef1968 ef1969 aspS ef2340 ef2341 ef2342 ef2343
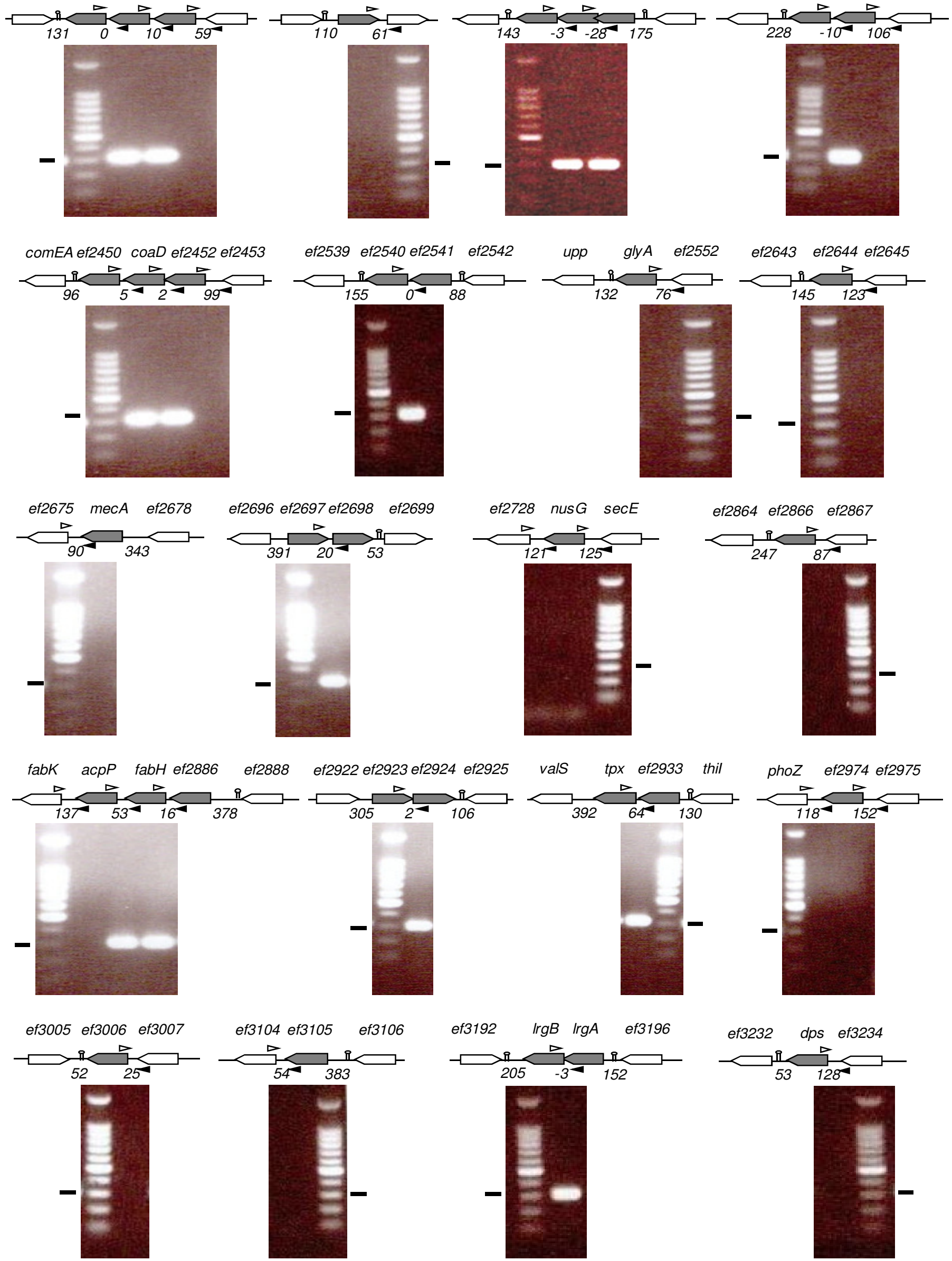
Figure S1 - C

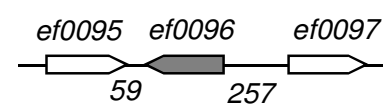

ef0081 ef0082 ef0083 ef0084

${ }_{367}^{\longrightarrow}$

$-\overbrace{57}^{\text {ef0194 gpm }} \overbrace{175}^{\text {ef0196 }}$

ef0251 ef0252 ef0253

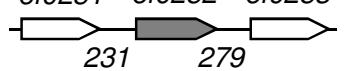

ef0439 ef0440 ef0441 ef0442 ef0443 ef0444

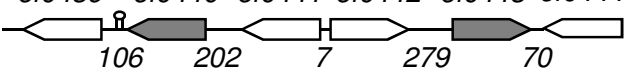

$\underbrace{e f 0696}_{189} \overbrace{88}^{\text {ef0697 ef0698 }}$

$\overbrace{171}^{\text {ef0708 }} \underbrace{p t s H}_{0} \underbrace{\text { ptsl ef0711 }}_{45}$

$\underbrace{e f 0780}_{234} u d \underbrace{e f 0782}_{805}$

ef0824 ef0825 ef0826

$\longrightarrow$

ef0906 ef0907 ef0908

$\succ_{244}^{\longrightarrow}$

$\underbrace{\text { luxS }}_{207} \underbrace{\text { asd }}_{34} \underbrace{\text { dapA rnjB }}_{0} \underbrace{\text { ef1186 }}_{45}$

ef1248 ef1249 ef1250

$156 \quad 208$

ef1257 ef1258 ef1259

$\longrightarrow \longrightarrow_{18}^{\longrightarrow}$

ef1269 ef1270 nusA

$\longrightarrow 206$

$\overbrace{216}^{\text {ef1503 lys }} \overbrace{140}^{\text {ef1505 }}$

ef1516 ef1518 ef1519 dnaG sigA

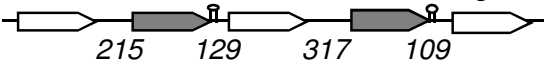

$\underbrace{\text { perR nox ef1587 ef1589 }}_{298}$

$\underbrace{\text { ef1684 hlyIlI }}_{123}$

$\overbrace{105}^{e f 1726 e b s A} \overbrace{101}^{e s b B}$

ef1738 ef1739 ef1740

181 
ef2034 ef2036 ef2037

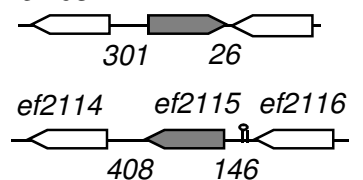

ef2212 ef2213 ef2214

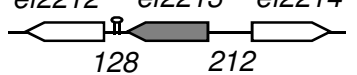

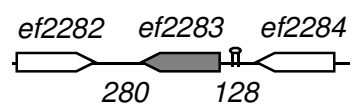

ef2290 ef2291 ef2292

$\overbrace{474}^{\longrightarrow 33}$

$\underbrace{e f 2442}_{211} \underbrace{r p s T}_{87}$

ef2546 ef2547 ef2548

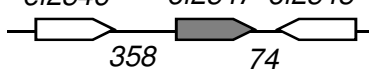

ef2678 trps ef2680

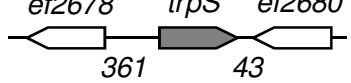

ef2832 ef2833 ef2834

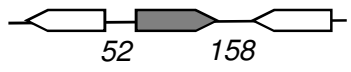

ef2855 rpmG-3 pbp2B

103126

ef2924 $\operatorname{csp} R \quad$ radC

$\overbrace{106}$ Michaux et al., 2012

$\underbrace{\text { thil }}_{159} \underbrace{\text { ef2935 ef2936 }}_{609}$

ef3051 ef3052 ef3053

$\overbrace{226}^{\longrightarrow}$

mreC ef3063 pnpA rpsO

$\longrightarrow \frac{187}{-67-26}$

ef3086 ef3087 ef3088

$182 \quad 297$

ef3115 rpmB ef3117

$\overbrace{274}^{\longrightarrow}$

ef3146 pgs $A$ ef3149

$\overbrace{546}^{\longrightarrow} \overbrace{125}^{i R}$

ef3176 ef3177 ef3178

$\longrightarrow_{164}^{\longrightarrow}$ 
Figure S1-D
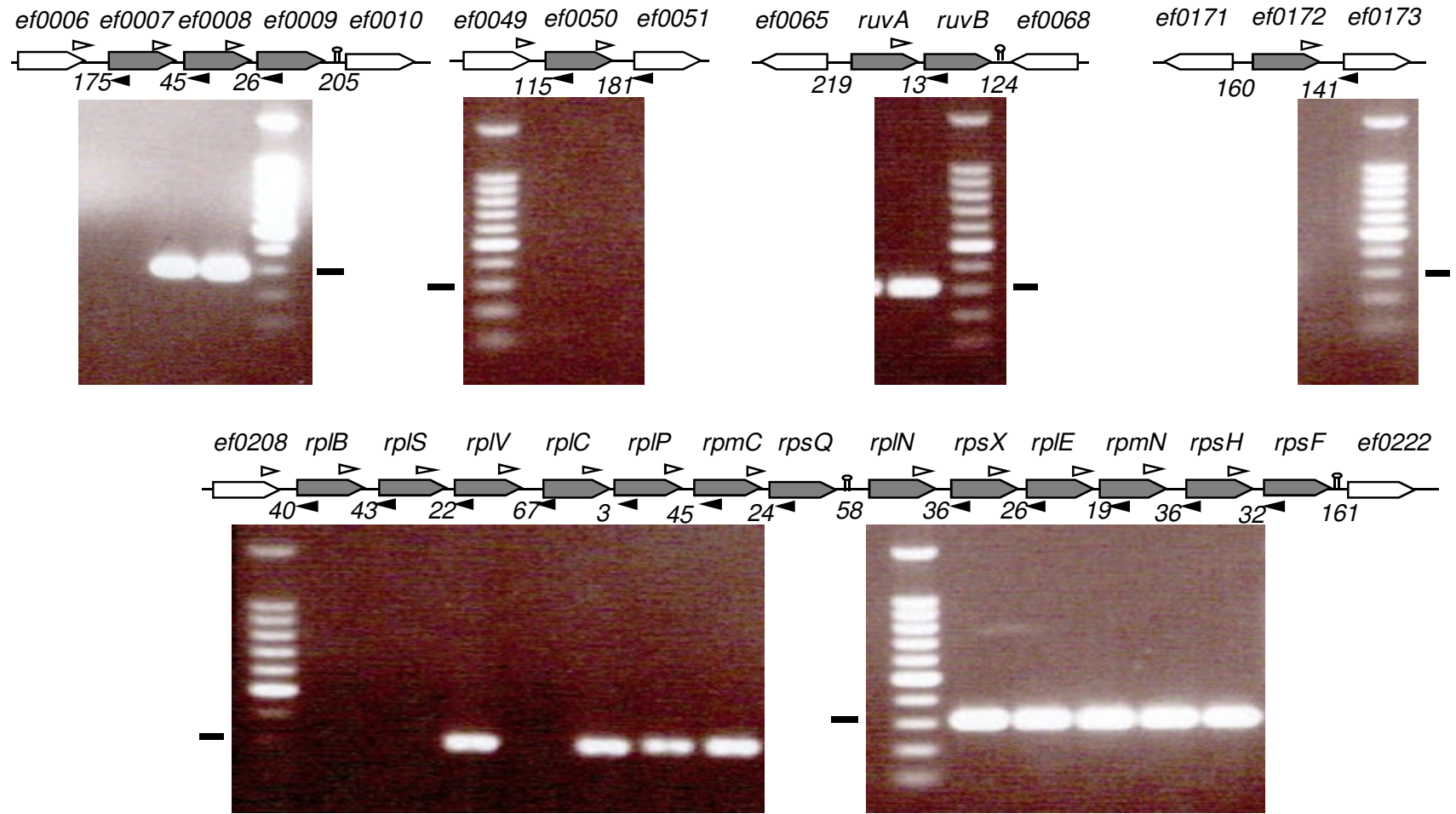
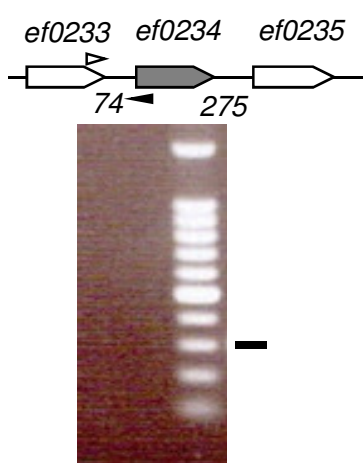

ef0364 ef0365 ef0366

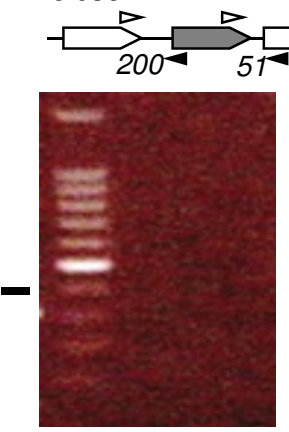

ef0759 ef0760 ef0761 ef0762
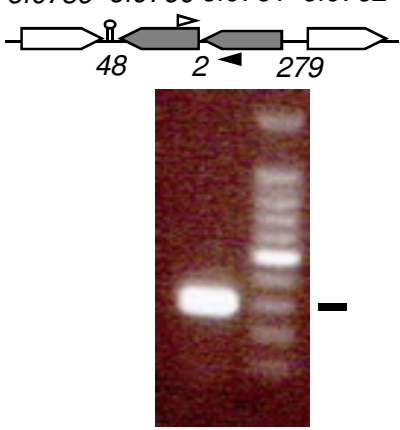
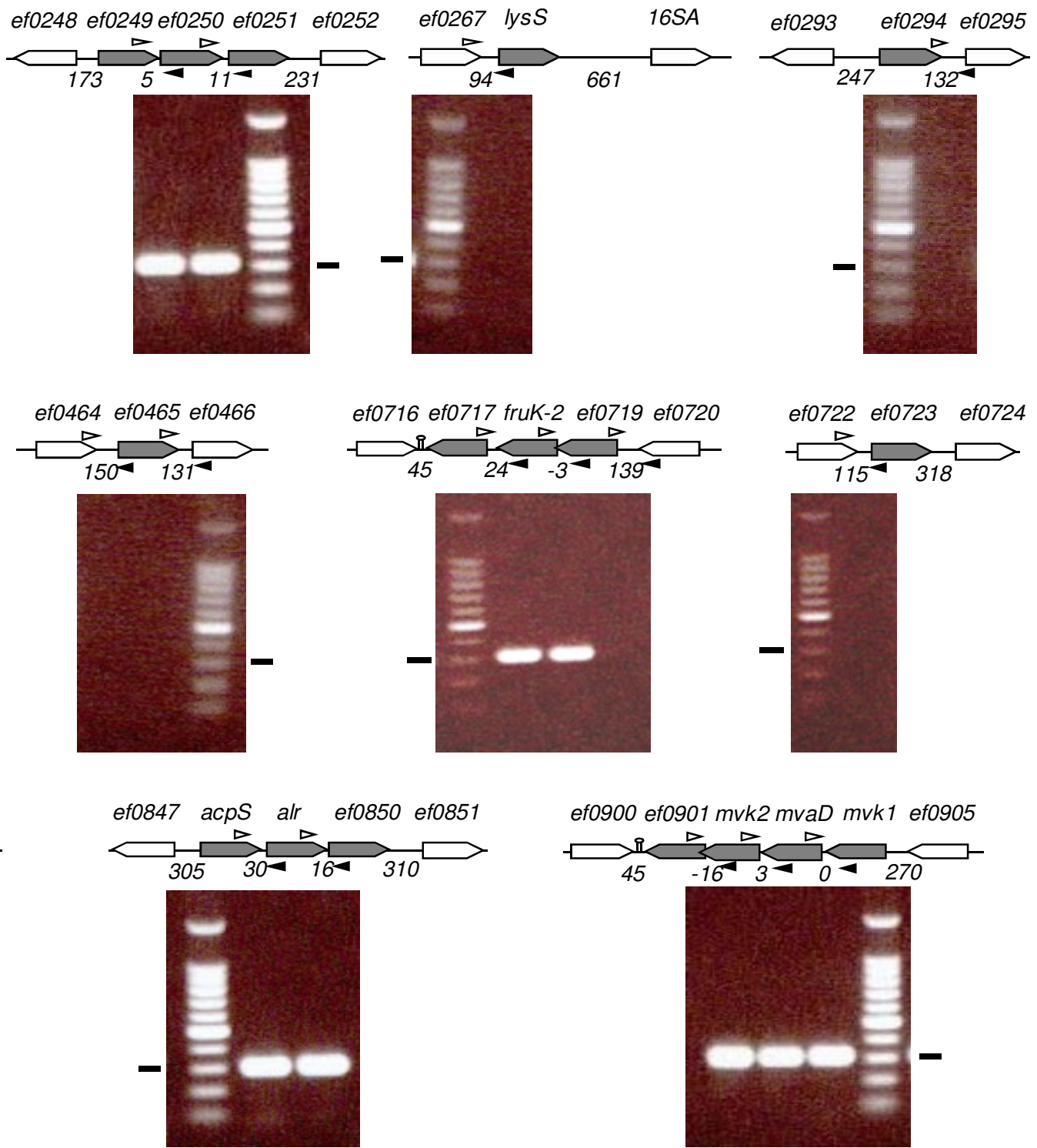

ef0900 ef0901 mvk2 mvaD mvk1 ef0905

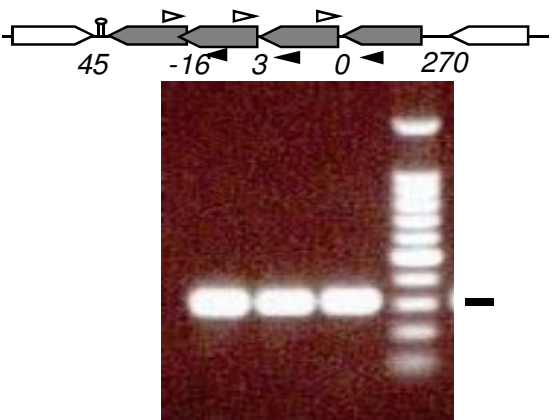



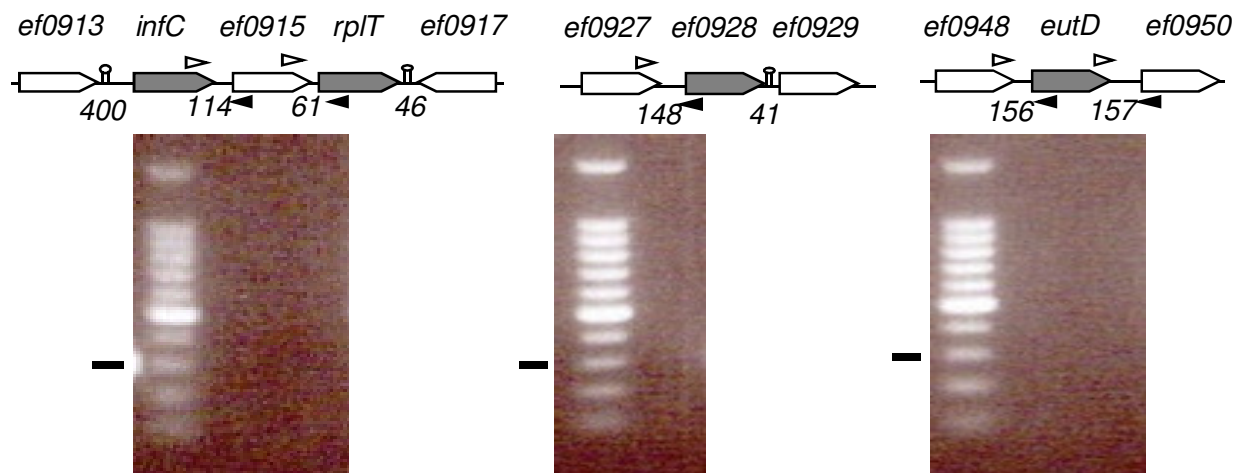

ef0986 ef0987 mraZmraW ef0990 pbpC mraY murD murG ftsQ fts A ef0997 ef0998 ef0999 ef1000
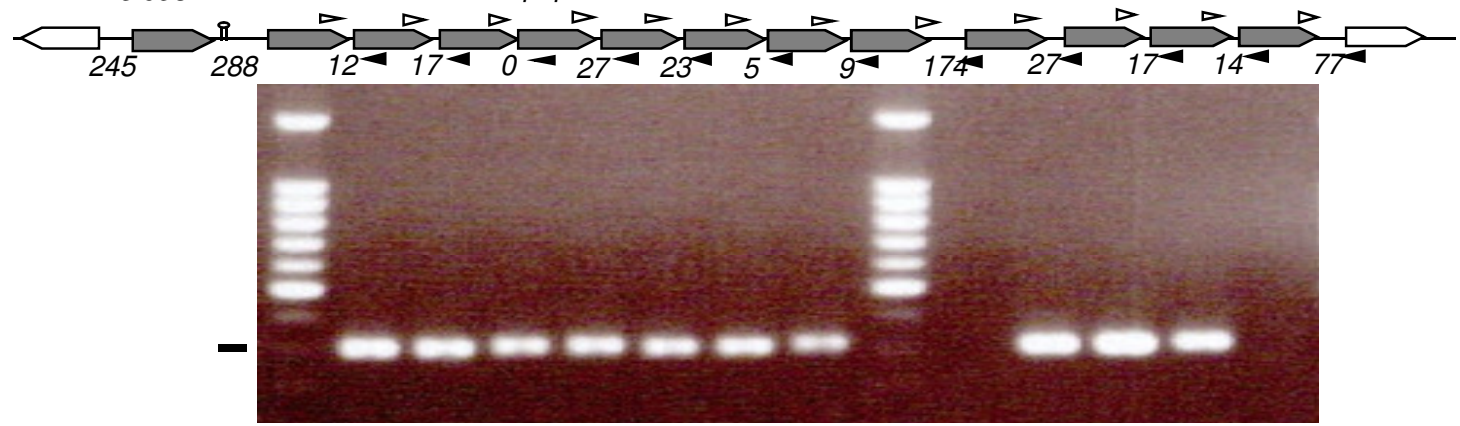

ef1044 pfk ef1046 ef1047 ef1048 ef1299 ef1300 ef1301 ef1302 gdhA pgi ef1417 ef1418 ef1419 ef1417
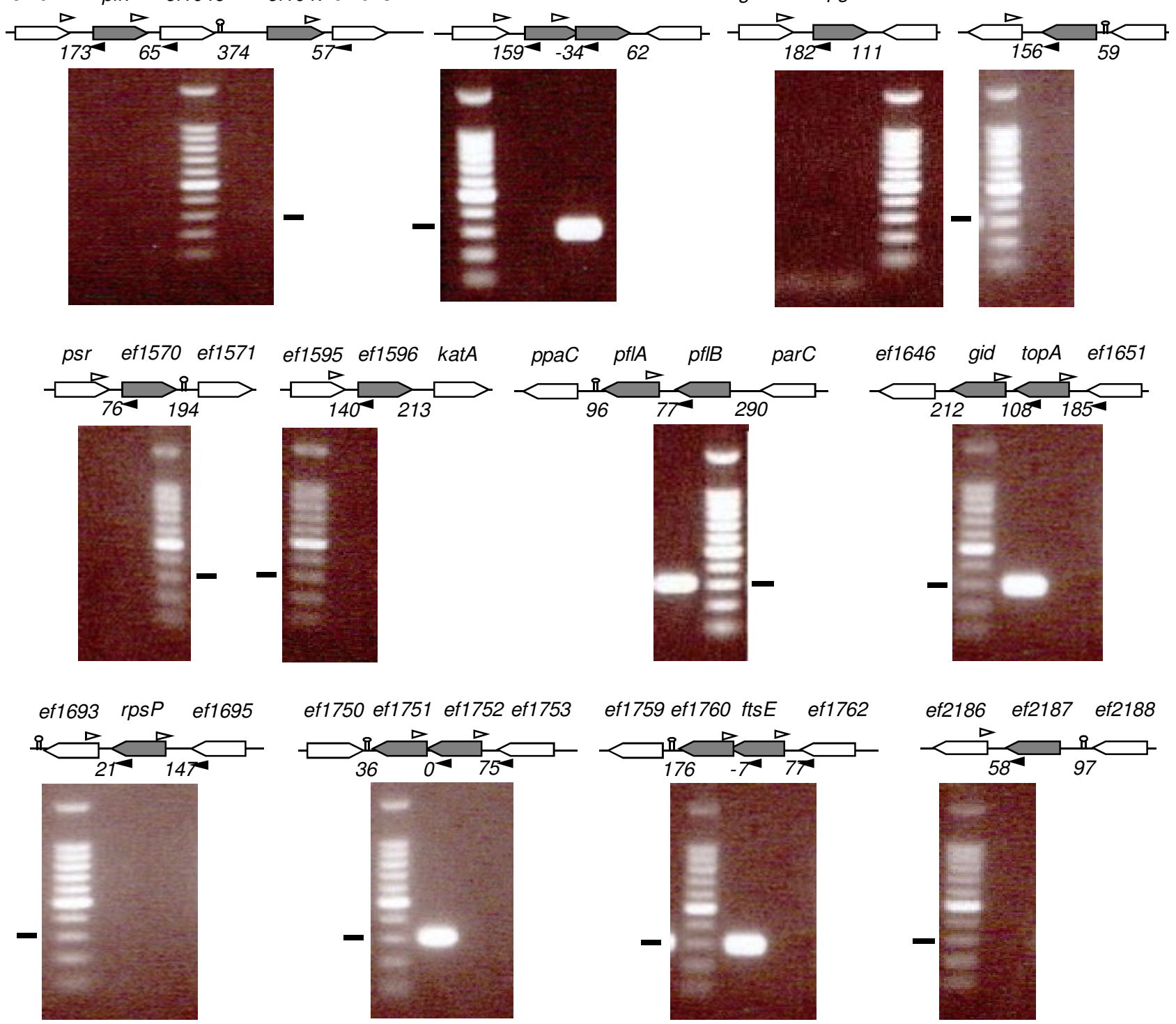

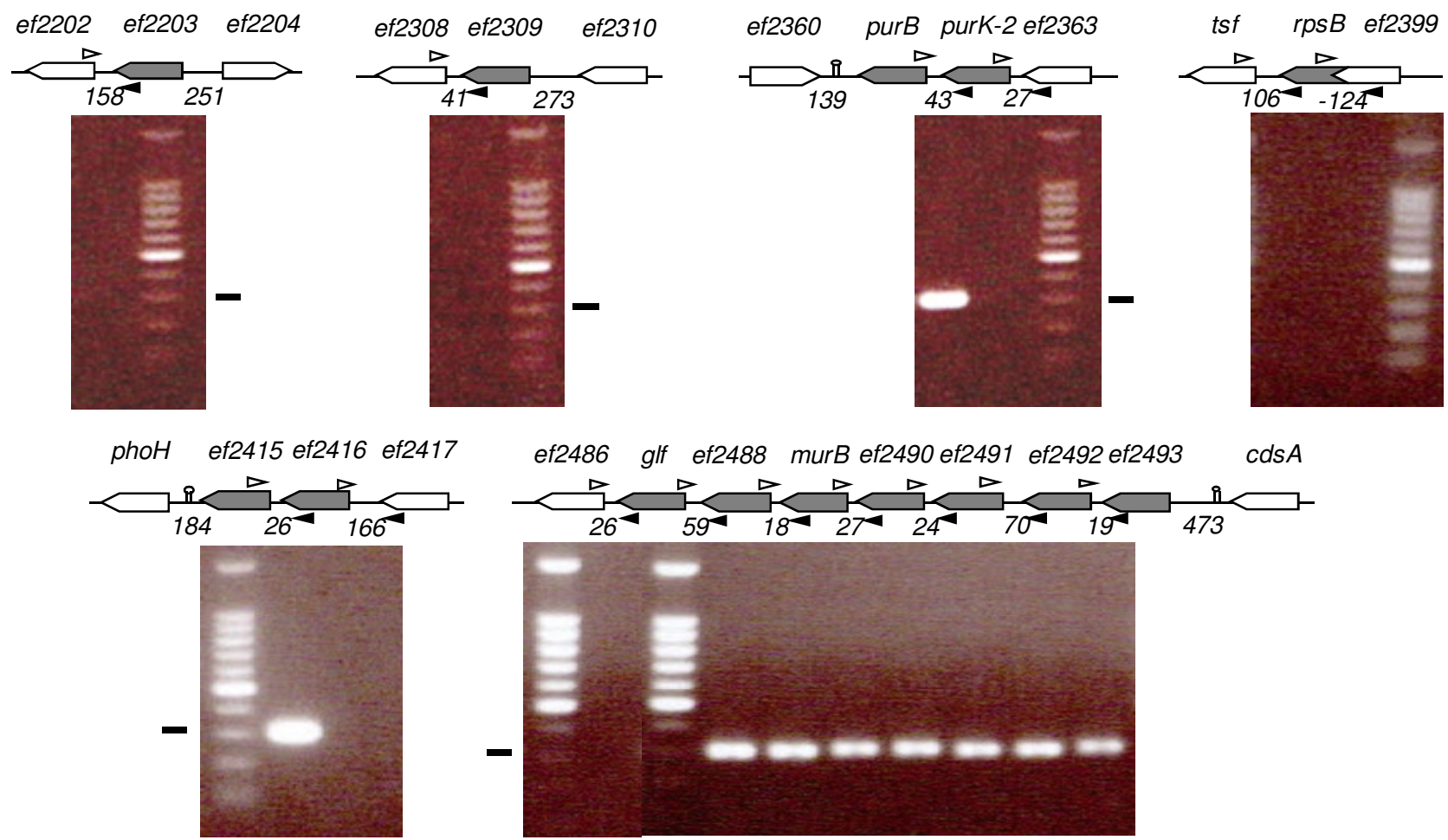

ef2499 ef2500 ef2501 ef2502 ef2503

ef2584 ef2585 ef2586 ef2587
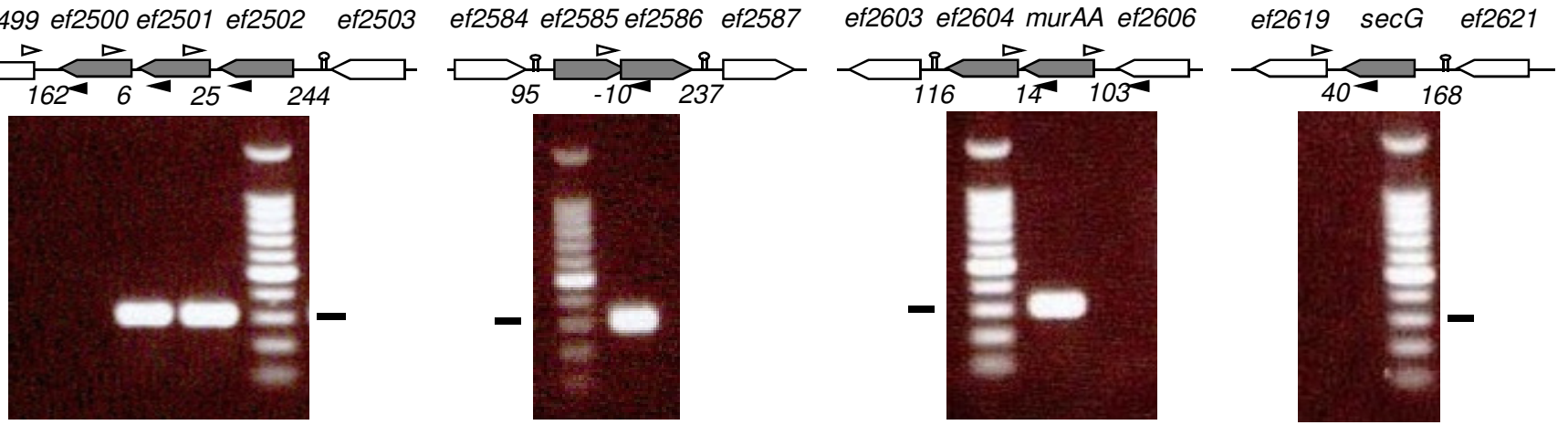

secE rpmG-2 ef2732

ef2792 ef2793 ef2794

ef2892 ef2893 ef2894

pepA ef3039 ef3040 ef3041
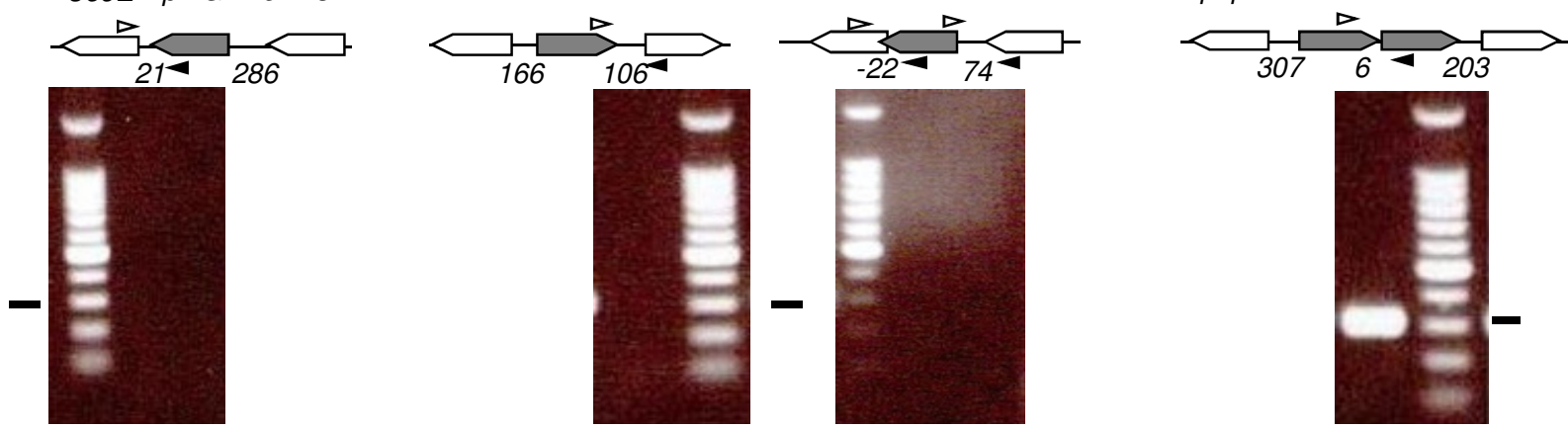

ef3105 ef3106 ef3107 ef3108 ef3109 ef3110 ef3111

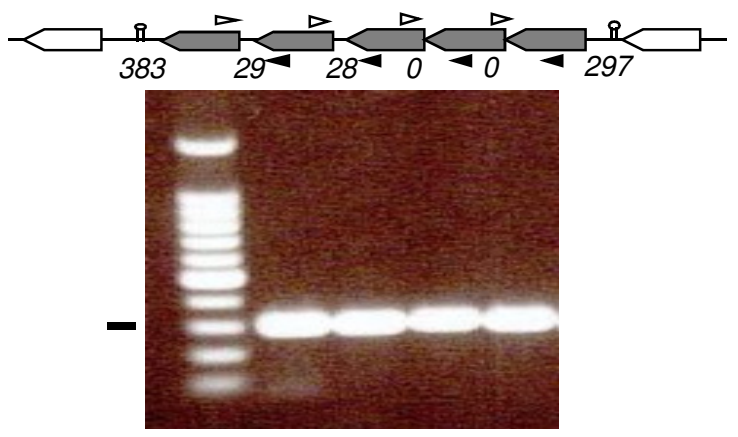

ef3209 ef3210 ef3211 ef3212 ef3213 ef3214
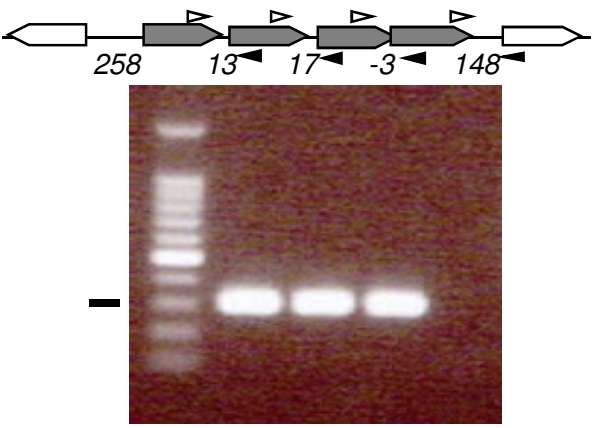


\section{Supplemental references}

Barcelona-Andrés B, Marina A, Rubio V (2002) Gene structure, organization, expression, and potential regulatory mechanisms of arginine catabolism in Enterococcus faecalis. J Bacteriol 184: 6289-6300.

Rana NF, Sauvageot N, Laplace J-M, Bao Y, Nes I, et al. (2013) Redox balance via lactate dehydrogenase is important for multiple stress resistance and virulence in Enterococcus faecalis. Infect Immun 81: 2662-2668. doi:10.1128/IAI.01299-12.

Rincé A, Giard JC, Pichereau V, Flahaut S, Auffray Y (2001) Identification and characterization of gsp65, an organic hydroperoxide resistance (ohr) gene encoding a general stress protein in Enterococcus faecalis. J Bacteriol 183: 1482-1488.

doi:10.1128/JB.183.4.1482-1488.2001.

Poyart C, Quesnes G, Trieu-Cuot P (2000) Sequencing the gene encoding manganesedependent superoxide dismutase for rapid species identification of enterococci. J Clin Microbiol 38: 415-418.

Garsin DA, Frank KL, Silanpää J, Ausubel FM, Hartke A, et al. (2014) Pathogenesis and Models of Enterococcal Infection. In: Gilmore MS, Clewell DB, Ike Y, Shankar N, editors. Enterococci: From Commensals to Leading Causes of Drug Resistant Infection. Boston: Massachusetts Eye and Ear Infirmary. Available: http://www.ncbi.nlm.nih.gov/books/NBK190426/. Accessed 25 June 2014.

Rincé A, Uguen M, Le Breton Y, Giard J-C, Flahaut S, et al. (2002) The Enterococcus faecalis gene encoding the novel general stress protein Gsp62. Microbiol Read Engl 148: $703-711$

Hancock L, Perego M (2002) Two-Component Signal Transduction in Enterococcus faecalis. J Bacteriol 184: 5819-5825. doi:10.1128/JB.184.21.5819-5825.2002.

Ross RP, Claiborne A (1997) Evidence for regulation of the NADH peroxidase gene (npr) from Enterococcus faecalis by OxyR. FEMS Microbiol Lett 151: 177-183.

Laport MS, Lemos JAC, Bastos Md M do CF, Burne RA, Giambiagi-De Marval M (2004) Transcriptional analysis of the groE and dnaK heat-shock operons of Enterococcus faecalis. Res Microbiol 155: 252-258. doi:10.1016/j.resmic.2004.02.002.

Bizzini A, Zhao C, Budin-Verneuil A, Sauvageot N, Giard J-C, et al. (2010) Glycerol is metabolized in a complex and strain-dependent manner in Enterococcus faecalis. $\mathrm{J}$ Bacteriol 192: 779-785. doi:10.1128/JB.00959-09.

Paulsen IT, Banerjei L, Myers GSA, Nelson KE, Seshadri R, et al. (2003) Role of mobile DNA in the evolution of vancomycin-resistant Enterococcus faecalis. Science 299: 20712074. doi:10.1126/science.1080613.

Verneuil N, Rincé A, Sanguinetti M, Auffray Y, Hartke A, et al. (2005) Implication of hypR in the virulence and oxidative stress response of Enterococcus faecalis. FEMS Microbiol Lett 252: 137-141. doi:10.1016/j.femsle.2005.08.043. 
Verneuil N, Rincé A, Sanguinetti M, Posteraro B, Fadda G, et al. (2005) Contribution of a PerR-like regulator to the oxidative-stress response and virulence of Enterococcus faecalis. Microbiol Read Engl 151: 3997-4004. doi:10.1099/mic.0.28325-0.

Low YL, Jakubovics NS, Flatman JC, Jenkinson HF, Smith AW (2003) Manganesedependent regulation of the endocarditis-associated virulence factor EfaA of Enterococcus faecalis. J Med Microbiol 52: 113-119.

Riboldi GP, Verli H, Frazzon J (2009) Structural studies of the Enterococcus faecalis SufU [Fe-S] cluster protein. BMC Biochem 10: 3. doi:10.1186/1471-2091-10-3.

Verneuil N, Sanguinetti M, Le Breton Y, Posteraro B, Fadda G, et al. (2004) Effects of the Enterococcus faecalis hypR gene encoding a new transcriptional regulator on oxidative stress response and intracellular survival within macrophages. Infect Immun 72: 4424-4431. doi:10.1128/IAI.72.8.4424-4431.2004.

Giard JC, Rince A, Capiaux H, Auffray Y, Hartke A (2000) Inactivation of the stress- and starvation-inducible gls24 operon has a pleiotrophic effect on cell morphology, stress sensitivity, and gene expression in Enterococcus faecalis. J Bacteriol 182: 4512-4520.

Deutscher J, Francke C, Postma PW (2006) How phosphotransferase system-related protein phosphorylation regulates carbohydrate metabolism in bacteria. Microbiol Mol Biol Rev MMBR 70: 939-1031. doi:10.1128/MMBR.00024-06.

Gao P, Pinkston KL, Nallapareddy SR, van Hoof A, Murray BE, et al. (2010) Enterococcus faecalis rnjB is required for pilin gene expression and biofilm formation. J Bacteriol 192: 5489-5498. doi:10.1128/JB.00725-10.

Michaux C, Martini C, Shioya K, Ahmed Lecheheb S, Budin-Verneuil A, et al. (2012) CspR, a cold shock RNA-binding protein involved in the long-term survival and the virulence of Enterococcus faecalis. J Bacteriol 194: 6900-6908. doi:10.1128/JB.01673-12. 